\title{
PREVENÇÃO SECUNDÁRIA À EVENTOS CARDIOVASCULARES: REVISÃO SISTEMÁTICA DE LITERATURA
}

\author{
Erik Ramos Silva*1, Paulo Sérgio Leahy Andrade Júnior², Mariane Costa Santos de Tavares ${ }^{3}$, \\ Cintia Maria Koenig4, Fernanda Cirilo Moura ${ }^{5}$, Guilherme Mendes Linhares ${ }^{5}$, Andressa Carlos de \\ Oliveira $^{5}$, Ana Clara de Sousa Araújo5, Tarcísio Nascimento Coutinho6, Bárbara Bernardes \\ Magalhães $^{6}$, Cauã Moreira Peixoto Pereira7, Milena Tofani Braga ${ }^{8}$ \\ and Lorraine Martins Dutra e Oliva ${ }^{9}$
}

\begin{abstract}
1Discente do Curso de Graduação em Medicina. Faculdades Santo Agostinho - FASA; ${ }^{2}$ Cardiologista Clínico e intervencionista. Docente do Curso de Graduação em Medicina. Faculdades Santo Agostinho - FASA; ${ }^{3}$ Fisioterapeuta. Discente do Curso de Graduação em Medicina. Faculdades Santo Agostinho - FASA; ${ }^{4}$ Médica, Especialista em Saúde Coletiva pela Universidade Federal da Bahia - UFBA; ${ }^{5}$ Discente do Curso de Graduação em Medicina. Faculdades Santo Agostinho - FASA; ${ }^{6}$ Discente do Curso de Graduação em Medicina.Universidade Estadual do Sudoeste da Bahia - UESB; ${ }^{7}$ Discente do Curso de Graduação em Medicina. UNIFTC; ${ }^{8}$ Discente do Curso de Graduação em Medicina. Faculdades Integradas do Norte de Minas - FUNORTE; ${ }^{9}$ Discente do Curso de Graduação em Medicina. Faculdade UNINOVE
\end{abstract}

\section{ARTICLE INFO}

\section{Article History:}

Received $22^{\text {nd }}$ January, 2021

Received in revised form

$14^{\text {th }}$ February, 2021

Accepted $17^{\text {th }}$ March, 2021

Published online $22^{\text {th }}$ April, 2021

\section{Key Words:}

Doenças Cardiovasculares.

Prevenção secundária. Dislipidemia.

*Corresponding author:

Erik Ramos Silva

\begin{abstract}
A doença cardiovascular aterosclerótica é responsável por cerca de metade da morbidade e mortalidade em todo o mundo. Dentre os fatores de risco temos o tabagismo, a hipertensão arterial, diabetes mellitus e a dislipidemia - alteração quantitativa das lipoproteínas plasmáticas caracterizada por aumento e/ou redução das partículas - como os mais relevantes. Nesse contexto, a prevenção secundária dos indivíduos que já se encontram com essa doença instalada ou que possuem histórico de eventos clínicos relacionado, como infarto agudo do miocárdio e acidente vascular encefálico, consiste em uma série de medidas farmacológicas e não farmacológicas que visam evitar ou minimizar um novo evento clínico cardiovascular. $\mathrm{O}$ objetivo do presente estudo foi abordar a prevenção secundária à eventos cardiovasculares tendo como foco o controle do perfil lipídico do paciente. O estudo constitui uma revisão sistemática de literatura realizada nas bases de dados indexadas na Medical LiteratureAnalysis and Retrieval System Online (MEDLINE), e Literatura Latino-americana e do Caribe em Ciências da Saúde (LILACS). Foram utilizados para busca na literatura os descritores em ciências da saúde (DECS): doenças cardiovasculares; prevenção secundária e dislipidemia. A elaboração do estudo seguiu os preceitos da metodologia PRISMA (Preferred Reporting Items for Systematic Reviews and Meta-Analyses). Evidenciou-se a importância do controle do perfil lipídico para prevenção secundária de eventos cardiovasculares. A avaliação de parâmetros, bem como o estabelecimento de metas deve ser realizada de forma individual, levando-se em consideração o quadro de saúde do paciente, risco cardiovascular, e opções terapêuticas. O acompanhamento e controle do perfil lipídico em pacientes cardiopatas deve se dar de forma longitudinal e contínua, para que seja possível uma efetiva prevenção secundária de eventos e complicações cardiovasculares.
\end{abstract}

Copyright $@$ (2021, Erik Ramos Silva et al. This is an open access article distributed under the Creative Commons Attribution License, which permits unrestricted use, distribution, and reproduction in any medium, provided the original work is properly cited.

Citation: Erik Ramos Silva, Paulo Sérgio Leahy Andrade Júnior, Mariane Costa Santos de Tavares, Cintia Maria Koenig, Fernanda Cirilo Moura et al., 2021. "Prevenção secundária à eventos cardiovasculares: revisão sistemática de literatura", International Journal of Development Research, 11, (04), 4613746140 .

\section{INTRODUÇÃO}

As doenças cardiovasculares (DCV) representam a principal causa de incapacitação e de mortalidade entre pessoas no Brasil e no mundo (GBD 2013 Mortality and Causes of Death Collaborators, 2015). Dados apontam que as DCV são as principais causas de morte em mulheres e homens no Brasil (Tufik, et al., 2017) (Figueiredo, Oliveira, Sanches, Matias, \& Radovanovic, 2018). Sendo responsáveis por cerca de $20 \%$ de todas as mortes em indivíduos acima de 30 anos. Tais doenças são um dos problemas de saúde pública mais relevantes da atualidade, tendo em vista o seu crescimento acelerado, sobretudo nos países em desenvolvimento, em que $80 \%$ dos óbitos se dá por doenças crônicas, particularmente as DCV(World Health Organization, 2020). Dentre os fatores de risco para desenvolvimento de DCV destacam-se a pressão arterial elevada, hiperglicemia, quadro dislipidêmico(alteração quantitativa das lipoproteínas plasmáticas caracterizada por aumento e/ou redução das partículas), tabagismo,sedentarismo, sobrepeso e obesidade, além de 
comorbidades como o diabetes mellitus (DM)(Raymond, Rocco, \& Hazen, 2014)(Kummel, 2010). Considera-se um quadro dislipidêmico então a ocorrência de elevação dos níveis plasmáticos de colesterol de baixa densidade (LDL -c), aumento de triglicérides (TG) e/ou redução dos níveis de colesterol de alta densidade (HDL -c).Desta forma, de acordo com o tipo de alteração dos níveis séricos de lipídeos, a dislipidemia pode ser classificada como: hipercolesterolemia isolada, hipertrigliceridemia isolada, hiperlipidemia mista e HDL-C baixo (Barroso, et al., 2021). A abordagem adequada destes fatores de risco está associada a uma diminuição na incidência e na progressão das doenças cardiovasculares. As diferentes diretrizes sobre prevenção de dislipidemia e DCV existentes, embora tenham alguns pontos diversos, ressaltam a importância da estimativa de risco cardiovascular individual, e controle do quadro dislipidêmico para prevenção secundária de eventos cardiovasculares (Macedo \& Faerstein, 2017). Para que o paciente consiga se manter dentro da meta lipídica estabelecida faz-se necessária a adoção de uma série de medidas farmacológicas e não farmacológicas(Chen, et al., 2019)(Tufik, et al., 2017). O tratamento não medicamentoso, ainda baseia-se em terapia nutricional com dieta isenta de ácidos graxos trans, consumo de fibras solúveis, substituição parcial de ácidos graxos saturados por mono e poli-insaturados - como azeite de oliva, oleaginosas, alimentos da série ômega 6 . A isso soma-se mudanças de estilo de vida no controle das dislipidemias tais como: perda de peso, programa de atividade física regular e cessação do tabagismo (Sociedade Brasileira de Cardiologia - SBC, 2019). Desta forma, diante da grande influência da dislipidemia no desenvolvimento de complicações cardiovasculares e metabólicas, principalmente de pacientes cardiopatas, o objetivo do presente estudo foi abordar a prevenção secundária à DCV tendo como foco o controle do perfil lipídico do paciente.

\section{MÉTODOS}

Trata-se de uma revisão sistemática de literatura. O estudo foi estruturado conforme proposto na metodologia Preferred Reporting Items for Systematic Reviews and Meta-Analyses (PRISMA). A declaração PRISMA consiste em uma lista requisitos composta por 27 itens, e a utilização de um diagrama de fluxo para seleção dos estudos que irão compor à revisão sistemática. A busca qualificada foi realizada nas bases de dados indexadas na Medical LiteratureAnalysis and Retrieval System Online (MEDLINE), e Literatura Latinoamericana e do Caribe em Ciências da Saúde (LILACS). Foram utilizados para busca na literatura os descritores em ciências da saúde (DECS): doenças cardiovasculares; prevenção secundária e dislipidemia.Foram considerados somente artigos originais sobre prevenção secundária de eventos cardiovasculares através do controle de perfil lipídico, disponíveis integralmente, nos idiomas inglês, português e espanhol, publicados entre os anos de 2016 a 2021. A busca foi feita por dois pesquisadores independentes, visando maior acurácia na seleção dos estudos.

\section{RESULTADOS E DISCUSSÃO}

A análise de adequabilidade dos estudos seguiu a seleção dos títulos, análise dos resumos, e por fim, leitura completa destes, com análise qualitativa dos dados apresentados. No Fluxograma 1 está representada o processo de seleção dos estudos. Dados da Pesquisa Nacional de Saúde apontam que no Brasil a prevalência de colesterol total $\geq 200 \mathrm{mg} / \mathrm{dL}$ na população foi de $32,7 \%$, mais elevada em mulheres $(35,1 \%)$. As alterações lipídicas foram mais significativas em indivíduos com idade superior à 45 anos, e baixa escolaridade. Verificou-se ainda que a prevalência de HDL alterado esteve presente em $31,8 \%$ da população, sendo de $42,8 \%$ no sexo masculino e $22,0 \%$ no feminino (Malta, et al., 2019). Para avaliar o risco de DCV, é necessário realizar o lipidograma, que caracteriza-se como um conjunto de exames que precisam ser realizados frequentemente e em conjunto (Teixeira, et al., 2018). O perfil lipídico geral, segundo a Sociedade Brasileira de Análises Clínicas (2016), inclui: Colesterol total (CT), Colesterol HDL - com frequência chamado colesterol "bom" , Colesterol LDL - com frequência chamado colesterol "mau" e os Triglicerídeos. O resultado desses exames são ótimos indicadores de risco de Infarto do Miocárdio ou de Acidente Vascular Cerebral(Sociedade Brasileira de Análises Clínicas, 2016). Dosagens laboratoriais, são realizadas para avaliar três exames: Colesterol total, HDL-C e triglicerídeos. O LDL-C, pode ser aferido por dosagem direta ou estimado a partir da fórmula de Friedewald. Fórmula de Friedewald: $\mathrm{LDL}-\mathrm{C}=(\mathrm{CT}-[\mathrm{HDL}-\mathrm{C}+\mathrm{TG} / 5])$, se $\mathrm{TG}<400 \mathrm{mg} / \mathrm{dL}$. De acordo com estudo da Sociedade Brasileira de Pediatria (2017), nessa fórmula o jejum é necessário, o divisor dos triglicerídeos é fixo = "5"; e o LDL- -C não pode ser estimado se os triglicerídeos forem $>$ $400 \mathrm{mg} / \mathrm{dL}$. Isso faz com que os valores de LDL-C não sejam estimados caso os triglicerídeos sejam superiores a $400 \mathrm{mg} / \mathrm{dL}$, limitando a utilidade da fórmula; e requerendo a dosagem direta do LDL-C(Sociedade Brasileira de Análises Clínicas, 2016).

É consenso entre os estudos pesquisados que o controle do perfil lipídico representa uma das estratégias de maior relevância na prevenção secundária à eventos cardiovasculares(Catalina, et al., 2017). O descontrole lipídico configura-se como a base da patogênese aterosclerótica(Nayor \& Vasan, 2016). Na aterosclerose após lesão inicial do endotélio verifica-se acúmulode lipoproteínas de baixa densidade (LDLs) e LDL oxidadas (alteradas) na camada íntima do vaso. $\mathrm{O}$ processo inflamatório e obstrutivo subsequente constitui a principal etiologia de eventos cardiovasculares. Assim, associado à fatores genéticos, controle de demais doenças de base, e hábitos de vida, o controle lipídico é referido como primordial na prevenção secundária de DCV(Faludi, et al., 2017). Após a estratificação de risco de um paciente, estabelecem-se as metas terapêuticas primária e secundária a serem atingidas no tratamento da dislipidemia, de acordo com o nível de risco global presente (baixo, intermediário, alto e muito alto). A meta primária a ser atingidaé o valor recomendado do LDL-C e a secundária, o valor recomendado do colesterol não-HDL (lipoproteína de alta densidade), calculado pela subtração do HDL-C do colesterol total, representando a concentração de lipoproteínas aterogênicas no plasma(Barroso, et al., 2021)(Sociedade Brasileira de Cardiologia - SBC, 2019). Pacientes que já tiveram algum evento clínico cardiovascular, ou que possuem uma doença aterosclerótica significativa são classificados pacientes de "muito alto risco cardiovascular", visto que possuem maiores chances de recidivas e, portanto, devem adotar uma série de medidas farmacológicas e não farmacológicas mais rigorosas que visam evitar ou minimizar um novo evento clínico(Sociedade Brasileira de Cardiologia - SBC, 2019).

Pacientes que já tiveram eventos clínicos relacionados a DCV previamente têm chances muito maiores de terem recidiva ou progressão da doença e, devido a isso,devem seguir o protocolo da prevenção secundária, isto é, o tratamento deve ser mais intensivo, estabelecendo metas lipídicas mais rigorosas, como estabelece a Sociedade Brasileira de Cardiologia (2019). Nesses casos, a meta de LDL-c deve ser $<50 \mathrm{mg} / \mathrm{dl}$, e a meta do não HDL-c deve ser $<80$ $\mathrm{mg} / \mathrm{dL}$. Embora se reconheça a relação epidemiológica inversa entre níveis de HDL-c e incidência de DCV, os estudos de intervenção mais recentes falharam em demonstrar benefício clínico por meio de fármacos que elevem o HDL-c. Com relação aos triglicérides, considera-se que pacientes com valores $\geq 500 \mathrm{mg} / \mathrm{dL}$ devem receber terapia apropriada para redução do risco de pancreatite. Aqueles com valores entre 150 e $499 \mathrm{mg} / \mathrm{dL}$ devem receber terapia individualizada, com base no risco cardiovascular e nas condições associadas(Sociedade Brasileira de Cardiologia - SBC, 2019). No caso de pacientes classificados como alto risco, a meta de LDL-c (meta primária) deve ser $<50 \mathrm{mg} / \mathrm{dl}$, e a meta do não HDL-c (meta secundaria) deve ser $<80 \mathrm{mg} / \mathrm{dl}$. Embora se reconheça a relação epidemiológica inversa entre níveis de HDL- c e incidência de DCV, os estudos de intervenção mais recentes falharam em demonstrar benefício clínico por meio da elevação do HDL-c. Com relação aos triglicérides, considera-se que pacientes com valores $\geq 500 \mathrm{mg} / \mathrm{dL}$ devem receber terapia apropriada para redução do risco de pancreatite. Já aqueles com valores entre 150 e $499 \mathrm{mg} / \mathrm{dL}$ devem receber terapia individualizada, com base no risco cardiovascular e 
nas condições associadas(Sociedade Brasileira de Cardiologia - SBC, 2019) (Macedo \& Faerstein, 2017). Manter-se na meta lipídica estabelecida é de extrema importância para prevenção de agravos, como se pode observar, estudos caso-controle, observacionais e genéticos atestam o colesterol plasmático elevado como um dos principais fatores de risco modificáveis para DCV, principalmente para DAC, mas também para AVC isquêmico (Malta, et al., 2019).

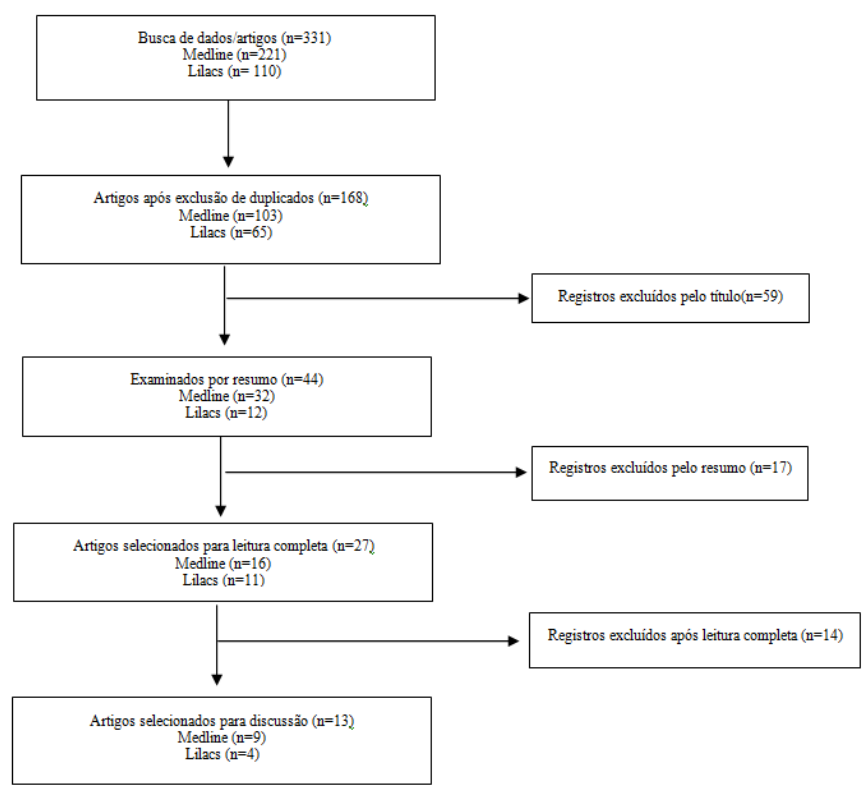

Fonte: Autoria própria (2021).

\section{Fluxograma 1. Triagem e seleção de estudos conforme metodologia PRISMA}

Diretrizes atuais recomendam a terapia com estatinas para controle lipídico em pacientes com risco cardiovascular de 10 anos $\geq 7,5 \%$. A necessidade de avaliação individualizada e estruturação de metas lipídicas adequadas a cada paciente também foram pontos ressaltados nos estudos (Nayor \& Vasan, 2016)(Raymond, Rocco, \& Hazen, 2014).

Estudos de intervenção, por sua vez, demonstram inequívoca diminuição da taxa de desfechos cardiovasculares proporcionada pela redução do colesterol plasmático, particularmente dos níveis de LDLc.. Grandes ensaios clínicos com estatinas demonstram que, quanto maior a redução absoluta do LDL-c, maior a redução do risco relativo de eventos cardiovasculares(Sociedade Brasileira de Cardiologia $\mathrm{SBC}$, 2019). São descritos como fatores de risco clássicos para complicações cardiovasculares o tabagismo, sedentarismo, quadro dislipidêmico, histórico familiar, além das comorbidades hipertensão, $\mathrm{DM}$ e obesidade. A presença de tais fatores deve nortear avaliação criteriosa do risco de doença cardiovascular, sobretudo doença arterial coronariana (DAC)(Précoma, et al., 2019). Em estudo realizado com 149 pacientes com idade entre 20-60 anos pode-seobservar a prevalência do HDL-Colesterolmenor que $40 \mathrm{mg} / \mathrm{dL}$ em 5 pacientes $(29,41 \%)$. Em $11 \%$ da amostra havia história familiar de doença cardiovascular prematura, e intrínseca associação entre dislipidemia, quadro de obesidade e sedentarismo(Bertoni, Zanardo, \& Ceni, 2017).A abordagem não farmacológica das dislipidemias é citada em diversos estudos como forma de prevenção secundária cardiovascular. A elaboração de uma dieta apropriada (Bertoni, Zanardo, \& Ceni, 2017), assim como a estruturação de uma rotina de atividades físicas devem compor o Plano Terapêutico dos indivíduos dislipidêmicos. Em estudo transversal com 24 indivíduos dislipidêmicos, e elevado risco cardiovascular verificou-se após nove meses de atividades físicas regulares a redução significativa do LDL-c e pequena diminuição de Triglicerídeos. A associação de hábitos de vida saudáveis, bem como estímulo ao protagonismo do indivíduo no autocuidado são de extrema importância, mesmo em casos cujo manejo farmacológico seja essencial(Eller, et al., 2017). Considerando a DAC a principal causa de morte prematura em todo o mundo. Em pacientes cardiopatas o controle dos fatores de risco cardiovasculares torna-se ainda mais desejável. Em estudo unicêntrico com pacientes com DAC incluindo 341 indivíduos somente e $5 \%$ e $34,9 \%$ dospacientes atingiram os níveis desejáveis de LDL-c e não-HDL-c, respectivamente após seis meses decorridos da revascularização miocárdica. Em um acompanhamento mediano de $4,33 \%$ a taxa de eventos cardiovasculares fatais e não fatais, eventos cardiovasculares adversos importantes (MACE - major adverse cardiovascular events) foi de $7,9 \%$, havendo associação significativa entre o baixo controle lipídico, e tais eventos. A taxa de reestenose foi de $0,9 \%$, a qual foisignificativamente influenciada por não-HDL-c médio. Diante dos resultados obtidos os autores ressaltam a necessidade de melhor controle do perfil lipídico apósprocedimentos de revascularização do miocárdio, mesmo em longo prazo, a fim de minimizar o risco de eventoscardiovasculares em pacientes com doenças coronarianas(Catalina, et al., 2017).

\section{CONSIDERAÇÕES FINAIS}

A associação entre quadros de dislipidemia e ocorrência de DCV já está bem estabelecida na literatura pesquisada. Em pacientes cardiopatas a DAC representa a principal causa de reintervenções, ou eventos mórbidos. Recomenda-se nestes pacientes, como prevenção secundária à eventos cardiovasculares avaliação periódica do risco cardiovascular, análise do perfil lipídico, assim como a promoção de hábitos de vida saudáveis. Estudar o perfil lipídico dos pacientes em prevenção secundária não é uma tarefa trabalhosa tendo em vista que os exames já são realizados de forma rotineira e esses devem ser encontrados no prontuário do paciente tanto no sistema público quanto no privado.Assim, a análise do perfil lipídico de pacientes cardiopatas em prevenção secundária é importante para possibilitar uma adoção de medidas mais rigorosas e eficazes na prevenção da doença.

\section{REFERÊNCIAS}

Barroso, W., Rodrigues, C., Bortolotto, L., Mota-Gomes, M., Brandão, A., Feitosa, A., \& Machado, C. (2021). Diretrizes Brasileiras de Hipertensão Arterial - 2020. Arq Bras Cardiol, 116(3), 516-658.

Bertoni, V., Zanardo, V., \& Ceni, G. (2017). Evaluation of lipid profile of patients with dyslipidemia of nutrition ambulatory specialty from URICEPP. Perspectiva, 22(126), 177-188.

Catalina, C., Adina, B., Smarandita, B., Angela, D., Dan, G., \& Silvia, M. (2017). Fatores Lipídicos de Risco Cardiovascular e Taxa de Eventos Cardiovasculares após Revascularização Miocárdica. International Journal of Cardiovascular Sciences, 30(1), 4-10.

Chen, Y., Freedman, N., Albert, P., Huxley, R., Shiels, M., Withrow, D., . . . González, A. (2019). Association of Cardiovascular Disease With Premature Mortality in the United States. JAMA Cardiol, 4(12), 1230-1238.

Eller, O., Diniz, A., Barros, D., Alves Júnior, E., Garcia, S., Alves, H., \& Queiroz, M. (2017). Controle das dislipidemias através do tratamento não farmacológico. Biofarm, 13(3), 25-30.

Faludi, A., Izar, M., Saraiva, J., Chacra, A., Bianco, H., Afiune Neto, A., . . L Lottenberg, A. (2017). Atualização da Diretriz Brasileira de Dislipidemias e Prevenção da Aterosclerose - 2017. Arq. Bras. Cardiol., 109(2), 1-76.

Figueiredo, F., Oliveira, R., Sanches, R., Matias, T., \& Radovanovic, C. (2018). Mortalidade por doenças cardiovasculares no Estado do Paraná. Cogitare Enfermagem, 23(4).

GBD 2013 Mortality and Causes of Death Collaborators. (2015). Global, regional and national age-sex specific all-cause and cause-specific mortality for 240 causes of death 1990-2013: a systematic analysis for the Global Burden of Disease Study 2013. Lancet, 385(9963), 117-171.

Kummel, D. (2010). Terapia Clínica e Nutricional na doença cardiovascular. Em L. Mahan , \& S. Stump, Alimentos, Nutrição e Dietoterapia (pp. 833-864). Rio de Janeiro: Elsevier. 
Macedo, L., \& Faerstein, E. (2017). Colesterol e a prevenção de eventos ateroscleróticos: limites de uma nova fronteira. Rev. Saúde Pública, 51(1), 5-7.

Malta, D., Szwarcwald, C., Machado, Í., Pereira, C., Figueiredo, A., Sá, A., . . . Rosenfeld, L. (2019). Prevalência de colesterol total e frações alterados na população adulta brasileira: Pesquisa Nacional de Saúde. Rev. bras. epidemiol., 22(2), E190005.

Nayor, M., \& Vasan, R. (2016). Recent Update to the US Cholesterol Treatment Guidelines: A Comparison With International Guidelines. Circulation, 133(18), 1795-1806.

Précoma, D., Oliveira, G., Simão, A., Dutra, O., Coelho, O., \& Izar, M. (2019). Atualização da Diretriz de Prevenção Cardiovascular da Sociedade Brasileira de Cardiologia - 2019. Arq. Bras. Cardiol., 113(4), 787-891.

Raymond, C., Rocco, L., \& Hazen, S. (2014). New cholesterol guidelines: worth the wait? Cleve Clin J Med, 81(1), 11-19.
Sociedade Brasileira de Análises Clínicas. (2016). Consenso Brasileiro para normatização da determinação laboratorial do perfil lipídico. Sociedade Brasileira de Análises Clínicas. Acesso em 22 de 02 de 2021, disponível em https://www.sbac.org.br/ wp-content/uploads/2017/09/ConsensoOficial_Perfil Lipidico 2016_v13.pdf

Sociedade Brasileira de Cardiologia - SBC. (2019). Atualização da Diretriz de Prevenção Cardiovascular da Sociedade Brasileira de Cardiologia - 2019. Arq. Bras. Cardiol., 113(04), 787-891.

Teixeira, R., Feijó, D., Valente, A., Carvalho, L., Granhen, H., Petroianu, A., \& Botelho, N. (2018). Influência da ooforectomia na glicemia e lipidograma. Acta Cir. Bras., 33(5), 415-419.

Tufik, S., Pires, G., Kim, L., Tempaku, P., Albuquerque, R., \& Andersen, M. (2017). Revisão sistemática sobre a epidemiologia das doenças cardiovasculares e respiratórias e suas associações com a poluição do ar em Vitória/ES . Clin. biomed. res , 37(02), 97-124.

World Health Organization. (2020). Doenças Cardiovasculares. Acesso em 01 de 03 de 2021, disponível em World Health Organization: https://www.who.int/health-topics/cardiovasculardiseases\#tab=tab_1 